\title{
The influencing factors and survival rate of pulmonary metastasis after liver resection :a retrospective cohort study
}

\author{
Yuting $\mathrm{He}^{1}$, Kunfu $\mathrm{Dai}^{1}$, and Haibo $\mathrm{Yu}^{1}$ \\ ${ }^{1}$ Henan Provincial People's Hospital
}

October 1,2020

\begin{abstract}
Background: Pulmonary metastasis(PM) form hepatocellular carcinoma(HCC) is a major indicator of a poor prognosis after liver resection. The study aimed to probe into the influencing factors for PM following open liver resection(OLR) and laparoscopic liver resection(LLR) with HCC. Method: All patients who suffered from LLR and OLR for primary HCC, who developed pulmonary metastasis, as first extrahepatic metastasis, were retrospectively analyzed in our department from January 2014 to August 2020. Results: Thirty-three cases were included (median age 50 years, $81.8 \%$ male). The patients with a single tumor had 23 cases $(69.7 \%)$, and a total of 10 patients $(30.3 \%)$ has two and more tumors. 21 cases (63.6\%) were smaller than $10 \mathrm{~cm}$ of tumor diameter. The median of operation time was 200 minutes (110-360), Portal vein occlusion occurred in 12 patients(36.4\%). Patients with intrahepatic recurrence received vertebroplasty combined chemotherapy(VPCC), targeted therapy, trans-arterial chemo-embolization(TACE) and radio frequency catheter ablation(RFCA) in $1(3 \%), 1(3 \%), 10(30.3 \%)$ and $7(12.1 \%)$ cases respectively. The 2 - and 3 - year cumulative probability of overall survival (OS) were $63 \%$ and $41 \%$. The median OS was 30.8 months. The median recurrence time was 6 months(1.7-4.8). The median of confirmed PM time from HCC was 12.5 months(1.5-61.1) and 17(51.5\%) patients died. Conclusion: Early detection of intrahepatic recurrence and treatment can prolong PM time and overall survival time.
\end{abstract}

\section{Introduction}

The changes experienced by the therapeutic method of HCC remain unprecedented over the past decade. LLR has become the main treatment for HCC since it first reported in 1991 with the development of endoscopic technology. Although the therapic technology of HCC has been improved continuously, its mortality is still high. Metastasis is the major lethal factor for postoperative patients of HCC. For metastasis, extrahepatic metastasis takes up 14.0-25.5\%[1]. The lung is the second common metastasis organ after LLR and OLR[2], meanwhile, the PM is accounting for 30-50\% of extrahepatic metastasis cases [3, 4].And it is difficult to detect PM early, other than regular imageological examination. Therefore, pulmonary metastasis impacts the postoperative survival rate of $\mathrm{HCC}[2]$. Through clinical comparison, there were more patients with pulmonary metastasis after LLR than OLR. The purpose of this study was to explore the effect factors of LLR and OLR for postoperative pulmonary metastasis.

\section{Materials and methods}

\section{Patients}

A total of 33 patients who suffered from $\operatorname{LLR}(n=6)$ and $\operatorname{OLR}(n=27)$ for primary HCC, who developed pulmonary metastasis, as first extrahepatic metastasis, were retrospectively analyzed in our department from January 2014 to August 2020.

The enhanced spiral computed tomography $(\mathrm{CT})$ as the diagnostic criteria of pulmonary metastasis was performed every 1 month during the first 6months and every 3 months thereafter. One case received chemother- 
apy and one patient received TACE to prevent or postpone postoperative recurrence of HCC. Other patients were reviewed regularly after surgery.

The patients who were pathological diagnosed with HCC accompanied by a history of liver resection were incorporated into the study. We eliminated the patients with the following criteria:(1) the surgical methods were conversion from laparoscopic to laparotomy and hand-assisted laparoscopic surgery;(2) first extrahepatic recurrence was not lung;(3) primary lung tumor;(4) had a history of invasive treatment; (5) incomplete case.

The date was assessed with preoperative factors(including age, sex, underline liver disease, Child-Pugh score, tumor size and number, portal vein thrombus),intraoperative factors(duration and number of portal vine occlusion, operation time),postoperative therapy(including chemotherapy, targeted therapy, radiofrequency ablation, TACE), laboratory inspection before PM(AFP, CA-199, neutrophil count, lymphocyte count, monocyte count, eosinophils count, basophil count), time of pulmonary metastasis and survival time and overall survival rate. The tumor size was defined as the maximum diameter of a single or the sum of maximum diameter of multiple tumors. We defined the time from the date of LLR or OLR to the date of death or last follow-up as survival time. The time of PM was the time from the date of LLR or OLR to the date of diagnosis of PM from HCC by CT.

\section{Statistical analysis}

We analyzed the corresponding continuous variables by the Mann-Whitney U-test, the $X^{2}$ test, Kaplan-Meier curve analysis, the log-rank test, multivariate Cox regression and other methods. Based on the unvaried analysis, variables with $P<0.05$ were included in the multivariate logistic regression analysis to determine the independent variables. All analyses were performed using SPSS 23.0 statistical software (SPSS, IL, USA). $P<0.05$ was considered statistically significant.

\section{Results}

In the study, thirty-three were included. Patients' basic features are shown in Table 1 . According to preoperative imaging material, the patients with a single tumor had 23 cases(69.7\%), and a total of 10 patients $(30.3 \%)$ has two and more tumors. $10 \mathrm{~cm}$ was defined as dividing point of tumor diameter, 21 cases $(63.6 \%)$ were smaller than $10 \mathrm{~cm}$, and 12 cases $(36.4 \%)$ were larger than $10 \mathrm{~cm}$. The median of operation time was $200 \min (110-360 \mathrm{~min}), 18 \operatorname{cases}(54.5 \%)$ of them were over 200min. Portal vein occlusion occurred in 12 patients(36.4\%), 8 cases $(24.2 \%)$ of them occluded once, 2 cases $(6.1 \%)$ occluded twice and 2 cases $(6.1 \%)$ occluded thrice. The boundary was15 min and $6 \operatorname{cases}(18.2)$ were greater than $15 \mathrm{~min}$. There were 15 $\operatorname{cases}(45.5 \%)$ of intrahepatic recurrence only. Patients with intrahepatic recurrence received vertebroplasty combined chemotherapy (VPCC), targeted therapy, TACE and radio frequency catheter ablation(RFCA) in $1(3 \%), 1(3 \%), 10(30.3 \%)$ and $7(12.1 \%)$ cases respectively. The median levels of AFP and CA-199 before PM were $77.12 \mathrm{ug} / \mathrm{L}(1.06-121000 \mathrm{ug} / \mathrm{L})$ and $29.05 \mathrm{ug} / \mathrm{L}(1.21-84.79 \mathrm{ug} / \mathrm{L})$ and greater than normal revel were $22(66.7 \%)$ and $18(54.5 \%)$ cases respectively. All patients with PM received chemotherapy until death or the end of the study. Each patient was presented with detailed information inTable 2 .

The 2- and 3- year cumulative probability of OS from LLR or OLR were $63 \%$ and $41 \%$. The median OS was 30.8 months $(95 \%$ CI 22.0-39.5) (Figure1a ). The median recurrence time was 6 months(1.7-4.8 months) after liver resection. The patients with intrahepatic recurrence who underwent OLR and LLR had higher OS than patients without intrahepatic recurrence $(\mathrm{P}=0.04)($ Figure1 $\mathbf{b})$. The $\mathrm{OS}$ of patients with intrahepatic recurrence who received VPCC, targeted therapy, TACE and RFCA was higher $(\mathrm{P}=0.03)$ ( Figure1 $\mathbf{c})$. The median of confirmed PM time from HCC was 12.5 months(1.5-61.1 months) and 17(51.5\%) patients died. Meanwhile, the OS of patients with late PM was significantly higher than that of patients with early PM time $(\mathrm{P}=0.02)$ ( Figure1 d $)$.

Table 1. Basic features of the 33 patients included in the study.

\begin{tabular}{ll}
\hline Features & Overall $(\mathbf{n}=\mathbf{3 3})$ \\
\hline Age, years & $50(26-70)$
\end{tabular}




\begin{tabular}{ll}
\hline Features & Overall(n=33) \\
\hline Male, N & $27(81.8 \%)$ \\
Female, N & $6(18.2 \%)$ \\
BMI, $\mathrm{kg} / \mathrm{m}^{2}$ & $25(18-33.5)$ \\
Smoke, N & $11(33.3 \%)$ \\
Drink, N & $12(36.4 \%)$ \\
Underlying disease, N & \\
Hypertension & $5(15.2 \%)$ \\
Diabetes & $8(24.2 \%)$ \\
Coronary heart disease & $2(6.1 \%)$ \\
Cirrhosis, N & $13(39.4 \%)$ \\
Liver disease virology, N & \\
HBV & $20(60.6 \%)$ \\
Tuberculosis & $3(9.1 \%)$ \\
Child-Pugh, N & \\
A & $30(90.9 \%)$ \\
B & $3(9.1 \%)$ \\
Operation method, N & \\
OLR & $27(81.8 \%)$ \\
LLR & $6(18.2 \%)$ \\
\hline
\end{tabular}

BMI, body mass index, HBV, hepatitis B virus, OLR, open liver resection, LLR, laparoscopic liver resection.

Table 2. Individual details and follow-up of the 33 patients included in the study.

\begin{tabular}{|c|c|c|c|c|c|c|c|c|c|c|c|c|}
\hline patient & $\begin{array}{ll} & \text { Number } \\
& \text { and } \\
\text { Sex } \quad \text { size of } \\
\text { age(yearst)umor }(\mathrm{cm}\end{array}$ & $\begin{array}{l}\text { Operatic } \\
\text { mmethod }\end{array}$ & $\begin{array}{l}\text { 10eratic } \\
\text { time (mir }\end{array}$ & $\begin{array}{l}\text { Duration } \\
\text { Num- } \\
\text { ber of } \\
\text { portal } \\
\text { orvine } \\
\text { nфcclusion }\end{array}$ & $\begin{array}{c}\text { AFP } \\
\text { n(mg்hlit) }\end{array}$ & $\begin{array}{l}\text { CA- } \\
199 \\
(\mathrm{ug} / \mathrm{l})\end{array}$ & $\begin{array}{l}\text { Neutroph } \\
\text { count } \\
\left(10^{9} / \mathrm{L}\right)\end{array}$ & $\begin{array}{l}\text { hillymphoc } \\
\text { count } \\
\left(10^{9} / \mathrm{L}\right)\end{array}$ & $\begin{array}{l}\text { coufonocyt } \\
\text { count } \\
\left(10^{9} / \mathrm{L}\right)\end{array}$ & $\begin{array}{l}\text { teosinoph } \\
\text { count } \\
\left(10^{9} / \mathrm{L}\right)\end{array}$ & $\begin{array}{l}\text { hPrasophi } \\
\text { count } \\
\left(10^{9} / \mathrm{L}\right)\end{array}$ & \\
\hline 1 & Male/43 3/14.4 & OLR & 210 & $0 / 0$ & 2250 & 17.9 & 6.03 & 0.52 & 0.97 & 0.02 & 0.01 & \\
\hline 2 & Female/42/0.4 & OLR & 305 & $1 / 15$ & 66583 & 25.21 & 2.3 & 0.53 & 0.42 & 0.05 & 0.01 & \\
\hline 3 & Female/48/3.6 & OLR & 130 & $0 / 0$ & 970.3 & 16.99 & 3.64 & 1.15 & 0.26 & 0.10 & 0.00 & \\
\hline 4 & Mela/45 2/8.4 & OLR & 240 & $1 / 11$ & 2.72 & 31.54 & 12.28 & 2.28 & 0.63 & 0.05 & 0.00 & \\
\hline 5 & Mela/51 1/4.5 & OLR & 180 & $0 / 0$ & 177.14 & 8.37 & 1.54 & 0.69 & 0.17 & 0.10 & 0.00 & \\
\hline 6 & Mela/53 1/8.5 & OLR & 230 & $0 / 0$ & 2.63 & 39.6 & 5.27 & 1.18 & 0.37 & 0.11 & 0.01 & \\
\hline 7 & Mala/38 1/9.8 & OLR & 245 & $1 / 13$ & 103.7 & 16.86 & 2.41 & 0.71 & 0.37 & 0.08 & 0.02 & \\
\hline 8 & Mala/50 1/13 & OLR & 220 & $0 / 0$ & 60.9 & 14.3 & 6.02 & 2.21 & 0.39 & 0.16 & 0.01 & \\
\hline 9 & Mala/38 1/3 & OLR & 135 & $0 / 0$ & 12.33 & 13.94 & 2.08 & 1.16 & 0.39 & 0.04 & 0.01 & \\
\hline 10 & Mela/51 1/7.2 & OLR & 270 & $0 / 0$ & 1154 & 10.7 & 3.93 & 1.09 & 0.49 & 0.17 & 0.03 & \\
\hline 11 & Mela/50 1/7.7 & LLR & 230 & $0 / 0$ & 5404 & 23.82 & 3.62 & 1.05 & 0.03 & 0.07 & 0.00 & \\
\hline 12 & Female/5D/9.3 & LLR & 140 & $0 / 0$ & 37.27 & 84.79 & 5.25 & 1.03 & 0.51 & 0.06 & 0.03 & \\
\hline 13 & Mela/65 1/2 & OLR & 110 & $0 / 0$ & 6.11 & 32.67 & 6.23 & 0.89 & 0.41 & 0.11 & 0.00 & \\
\hline 14 & Mela/45 1/7.7 & OLR & 140 & $0 / 0$ & 92.25 & 8.12 & 2.91 & 1.90 & 0.28 & 0.04 & 0.02 & \\
\hline 15 & Mala/54 1/6.7 & OLR & 255 & $0 / 0$ & 3.79 & 40.36 & 3.91 & 1.26 & 0.23 & 0.12 & 0.02 & \\
\hline
\end{tabular}




\begin{tabular}{|c|c|c|c|c|c|c|c|c|c|c|c|c|}
\hline patient & $\begin{array}{ll} & \text { Number } \\
& \text { and } \\
\text { Sex } \quad \text { size of } \\
\text { age(yearst)umor }(\mathrm{cn}\end{array}$ & $\begin{array}{l}\text { Operatio } \\
\text { mmethod }\end{array}$ & $\begin{array}{l}\text { o10perat } \\
\text { time }(\mathrm{m}\end{array}$ & $\begin{array}{l}\text { Duration } \\
\text { Num- } \\
\text { ber of } \\
\text { portal } \\
\text { orvine } \\
\text { in } \phi \text { cclusion }\end{array}$ & $\begin{array}{c}\text { AFP } \\
\text { n(mgihlil) }\end{array}$ & $\begin{array}{l}\text { CA- } \\
199 \\
(\mathrm{ug} / \mathrm{l})\end{array}$ & $\begin{array}{l}\text { Neutrop } \\
\text { count } \\
\left(10^{9} / \mathrm{L}\right)\end{array}$ & $\begin{array}{l}\text { countymphor } \\
\text { count } \\
\left(10^{9} / \mathrm{L}\right)\end{array}$ & $\begin{array}{l}\text { cytenocy } \\
\text { count } \\
\left(10^{9} / \mathrm{L}\right)\end{array}$ & $\begin{array}{l}\text { teosinoph } \\
\text { count } \\
\left(10^{9} / \mathrm{L}\right)\end{array}$ & $\begin{array}{l}\text { hilbasophil } \\
\text { count } \\
\left(10^{9} / \mathrm{L}\right)\end{array}$ & $\begin{array}{l}\text { Intre } \\
\text { met: } \\
\text { tasis } \\
\text { and }\end{array}$ \\
\hline 16 & Mela/50 1/12.7 & OLR & 240 & $0 / 0$ & 58.9 & 15.2 & 5.73 & 2.41 & 0.42 & 0.18 & 0.02 & \\
\hline 17 & Mela/47 1/10.7 & OLR & 194 & $1 / 18$ & 75.89 & 34.03 & 2.75 & 1.28 & 0.27 & 0.05 & 0.00 & \\
\hline 18 & Female/5 $\mathbb{\$} / 13.4$ & OLR & 185 & $0 / 0$ & 181.69 & 62.32 & 2.29 & 0.09 & 0.46 & 0.17 & 0.01 & \\
\hline 19 & Male/26 1/3.8 & LLR & 125 & $1 / 17$ & 3.22 & 2.00 & 2.91 & 1.33 & 0.13 & 0.08 & 0.00 & \\
\hline 20 & Male/54 4/9.1 & OLR & 328 & $0 / 0$ & 154.9 & 3.22 & 4.68 & 1.3 & 0.36 & 0.18 & 0.00 & \\
\hline 21 & Mela/70 1/6.4 & OLR & 195 & $0 / 0$ & 630.8 & 38.43 & 5.58 & 1.19 & 0.29 & 0.12 & 0.01 & \\
\hline 22 & Mela/64 1/6.1 & OLR & 360 & $0 / 0$ & 212.7 & 75.64 & 2.15 & 0.94 & 0.44 & 0.1 & 0.01 & \\
\hline 23 & Mela/53 1/12.3 & OLR & 309 & $3 / 25$ & 15764 & 69.3 & 4.11 & 1.15 & 0.36 & 0.28 & 0.03 & \\
\hline 24 & Mela/44 1/7 & OLR & 200 & $0 / 0$ & 169.6 & 12.7 & 2.67 & 1.08 & 0.42 & 0.08 & 0.02 & \\
\hline 25 & Mela/47 1/12 & OLR & 210 & $1 / 15$ & 77.12 & 35.91 & 2.31 & 1.17 & 0.29 & 0.15 & 0.00 & \\
\hline 26 & Mela/52 3/11.1 & LLR & 195 & $2 / 26$ & 2.5 & 29.09 & 3.37 & 2.58 & 0.41 & 0.12 & 0.00 & \\
\hline 27 & Mela/52 2/12 & LLR & 210 & $2 / 25$ & 21.4 & 29.9 & 2.91 & 1.86 & 0.33 & 0.21 & 0.02 & \\
\hline 28 & Mela/66 1/6.4 & OLR & 200 & $3 / 30$ & 1.78 & 53.1 & 4.19 & 1.95 & 0.24 & 0.04 & 0.00 & \\
\hline 29 & Mela/38 1/8.4 & OLR & 187 & $1 / 6$ & 3.16 & 29.6 & 3.21 & 1.37 & 0.65 & 0.09 & 0.02 & 100 \\
\hline 30 & Mela/49 2/4.5 & LLR & 190 & $0 / 0$ & 3.97 & 43.58 & 2.47 & 0.63 & 0.16 & 0.19 & 0.00 & \\
\hline 31 & Female/60/13.4 & OLR & 185 & $0 / 0$ & 1503 & 69.4 & 2.63 & 1.64 & 0.41 & 0.22 & 0.02 & 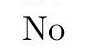 \\
\hline 32 & Female/6D/10.1 & OLR & 290 & $0 / 0$ & 121000 & 72.1 & 2.08 & 0.62 & 0.33 & 0.03 & 0.03 & T \\
\hline 33 & Mela/49 3/10.4 & OLR & 175 & $1 / 8$ & 1.06 & 1.21 & 2.63 & 1.22 & 0.35 & 0.01 & 0.01 & \\
\hline
\end{tabular}

VPCC, vertebroplasty combined chemotherapy, TACE, trans-arterial chemo-embolization ,RFCA, radio frequency catheter ablation, 

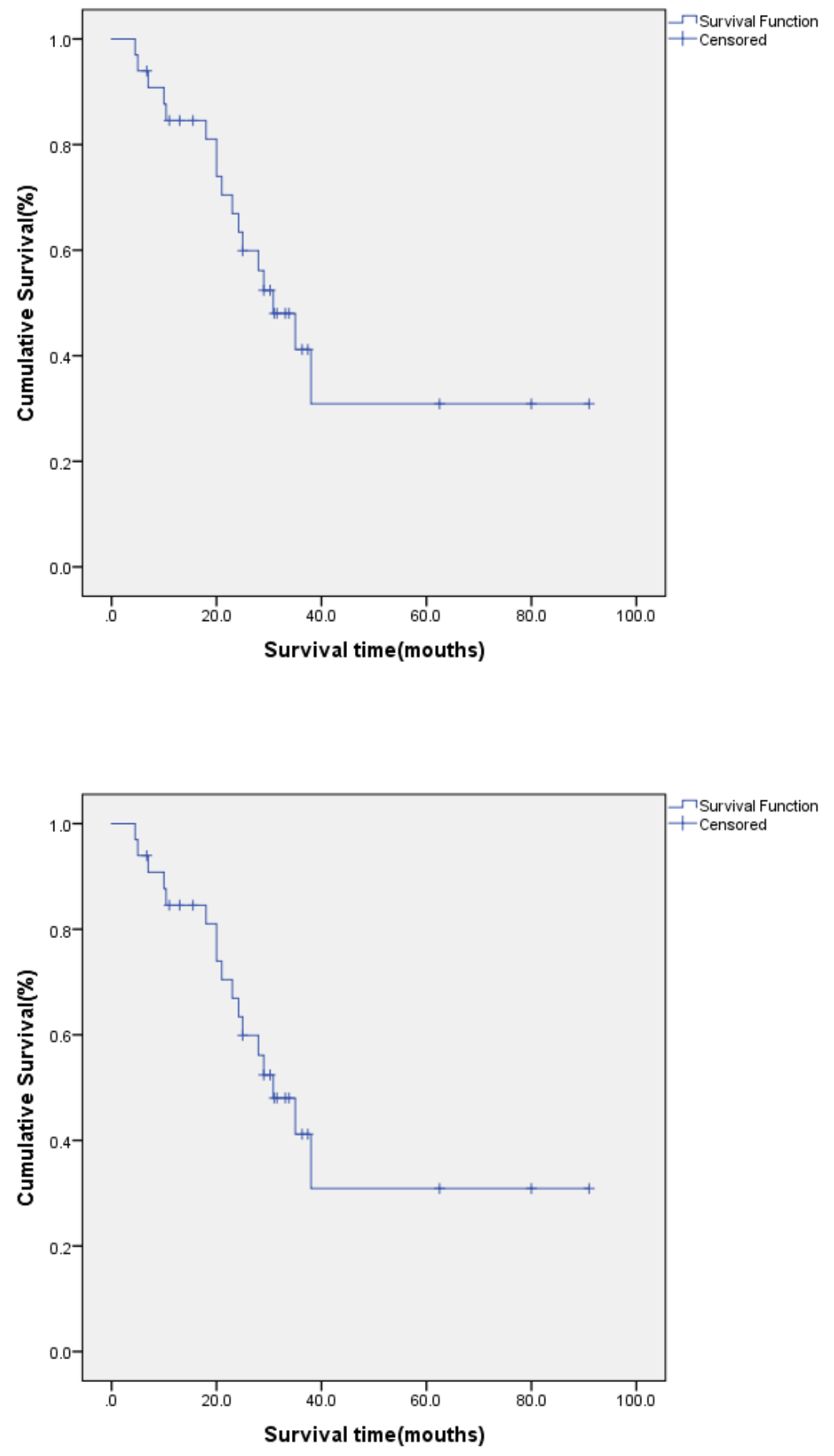

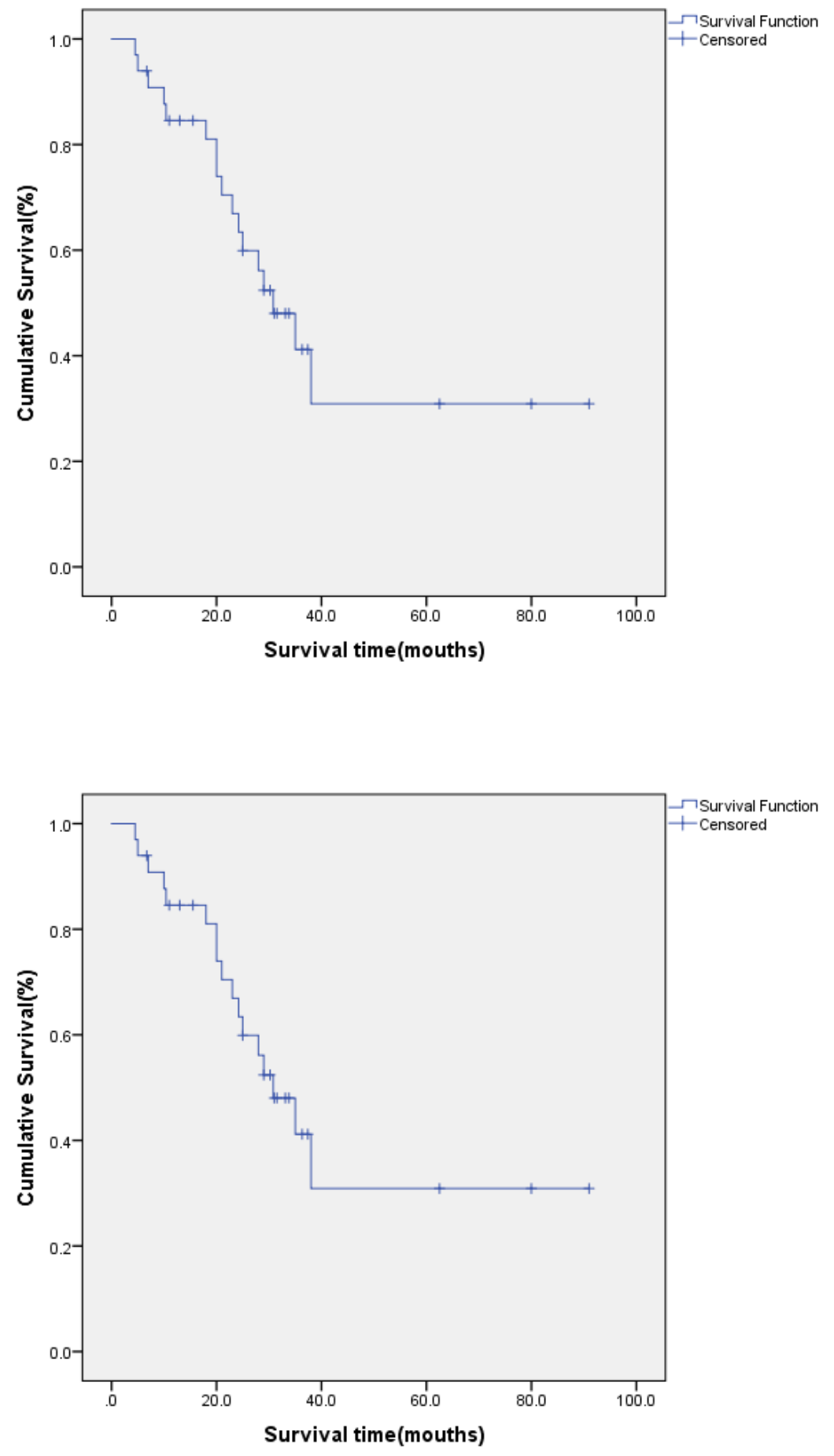


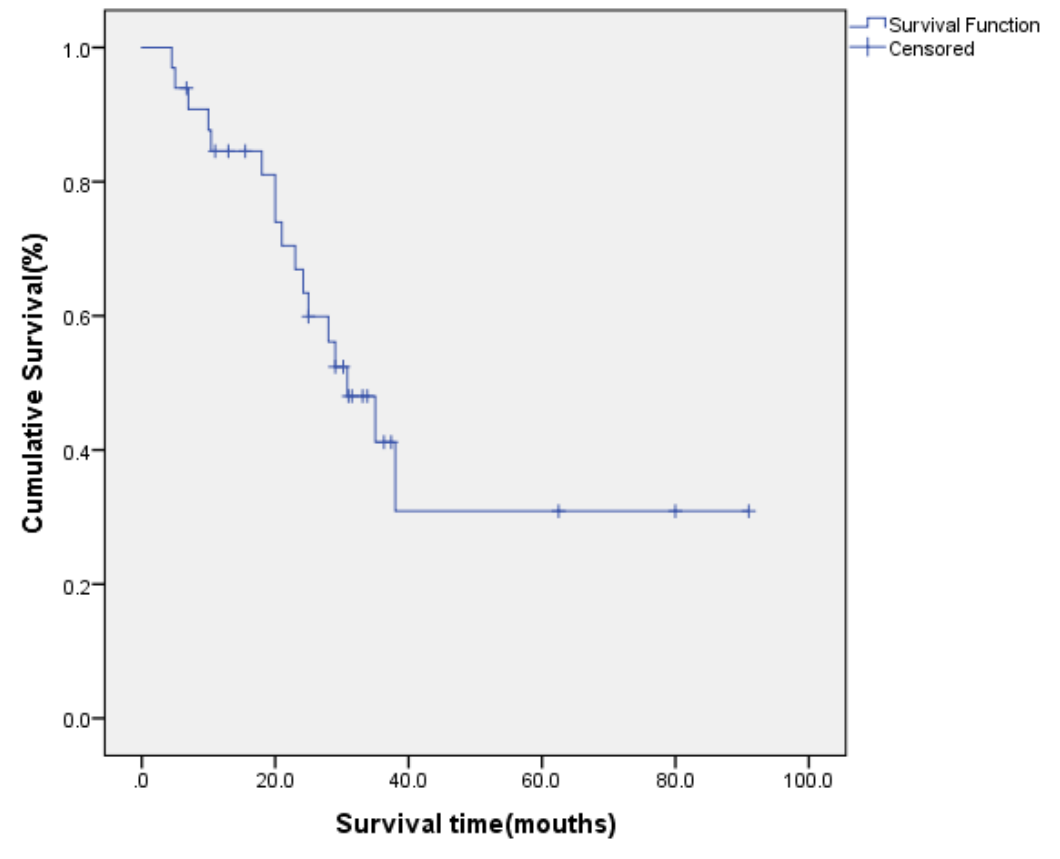

Figure1,a,2 and 3 cumulative survival of 33 patients included in the study with hepatocellular carcinoma following laparoscopic liver resection and open liver resection. b, Kaplan-Meier curve showing overall survival in patients with intrahepatic recurrence following laparoscopic liver resection and open liver resection. c, Kaplan-Meier curve showing overall survival in patients who with intrahepatic recurrence received vertebroplasty combined chemotherapy, targeted therapy, trans-arterial chemo-embolization and radio frequency catheter ablation after laparoscopic liver resection and open liver resection. d, Kaplan-Meier curve showing overall survival in patients with pulmonary metastasis from hepatocellular carcinoma who underwent laparoscopic liver resection and open liver resection.

\section{Discussion}

HCC is currently the fifth most malignant tumor and one of the main factors leading to tumor-related death $[1,5]$. According to the existing guidelines and standards of diagnosis and treatment of HCC from all over the world, although systemic chemotherapy, target therapy, TACE, RFCA and so on have been used to treat HCC, liver resection is the main treatment. With the continuous improvement of laparoscopic technology, the treatment of HCC has gradually transferred to a treatment with laparoscopic liver resection, radiofrequency ablation, and local chemotherapy as a supplement. However, the OS of patients undergoing liver resection for HCC has not significantly improved. According to related reports, HCC recurrence after liver resection is main factor to inhibit OS, including intrahepatic recurrence and extrahepatic metastasis $[6$, 7].

Intrahepatic recurrence is the most important factor affecting the survival of patients after liver resection. This study found that patients with intrahepatic recurrence after liver resection have higher OS than those without intrahepatic recurrence $(\mathrm{P}=0.04)$ (Figure 1b), and patients with intrahepatic metastases received TACE and RFCA, the higher the number of local treatment, the patient's OS was significantly prolonged $(\mathrm{P}=0.03)$ (figure 1c $)$. With the continuous improvement of imaging technology, tumor recurrence can be diagnosed early, and timely symptomatic treatment can be given, which reduces the possibility of tumor cell replication and metastasis in the liver after OLR and LLR, and prolongs the survival time.

This study is to investigate the influential factors of PM in HCC recurrence after OLR and LLR. A large 
number of articles have shown that PM from HCC is a major factor affecting the OS of patients after liver resection, in addition to intrahepatic recurrence [1]. And it has been pointed out that 1-year survival as low as $25 \%$ for extrahepatic metastasis after liver resection [8]. We observed that with 1-year and 2-years as the time nodes of PM, accompanied by the prolongation of PM time, the patient's OS also prolonged $(\mathrm{P}=0.02)$ (Figure 1d ). Combined with the analysis of the research results, patients undergoing OLR and LLR should be reviewed regularly according to the treatment guidelines to find out whether tumor recurrence is found in time, and timely intervention. It is necessary to preventive systemic chemotherapy and TACE to reduce the risk of postoperative intrahepatic recurrence and indirectly reduce postoperative PM is possible.

Some related reports pointed out that the risk of PM can be predicted by laboratory test indicators such as AFP and the ratio of neutrophils to lymphocytes and so on $[2,9,10]$. However, in this study, AFP, neutrophils and lymphocytes and PM were analyzed. The relevance is not significant, and needs further study. Moreover, the surgical method of liver resection and intraoperative portal vein occlusion have no statistically significant effect on PM. LLR or OLR has no effect on the survival time of patients after surgery. LLR has advantages in terms of reducing operating time, length of hospital stays, and postoperative complications [11-14]. LLR is recommended if there is no special requirement and the surgical indication is suitable. The median time of intrahepatic recurrence is 6 months as the time point to monitor the patient's intrahepatic recurrence, so as to detect and reduce the risk of PM in time.

\section{Limitations}

First, this paper is retrospective study, which has its limitation and deviation, like the development of surgical instruments, the improvement of physician's technique and so on. Second, the date was small and the results may exist occasionality.

\section{Conclusion}

Early detection of intrahepatic recurrence and timely intervention can delay the time of PM and prolong overall survival after liver resection for HCC. If necessary, chemotherapy or TACE can be used to prevent intrahepatic recurrence.

\section{Funding}

Grant supported this study from Henan medical science and technology project (201502018) and Henan medical science and technology project (2018020412).

\section{Availability of data and materials}

Please contact the corresponding author with a request for data and materials.

\section{Authors contributions}

This study was designed by Haibo Yu and Yuting He. Kungfu Dai conducted statistical analysis and explained the data. Haibo Yu and Yuting He wrote the manuscript. All authors reviewed and accepted the final manuscript.

\section{Ethics approval and consent to participate}

The Institutional Review approved this study Boards of Henan Provincial People's Hospital, (2015) Ethical Review No. (27). The research was conducted in accordance with the principals of the 1964 Declaration of Helsinki and its later amendments.

\section{Consent for publication}

Not applicable.

\section{Competing interests}

The authors declare that they have no competing interests. 


\section{Author details}

1.Department of Hepatobiliary Surgery, Henan Provincial People's Hospital, Zhengzhou, China;

\section{Reference}

1. Li J, Liu Y, Yan Z et al. A nomogram predicting pulmonary metastasis of hepatocellular carcinoma following partial hepatectomy. Br J Cancer 2014; 110: 1110-1117.

2. Wang L, Ye G, Zhan C et al. Clinical Factors Predictive of a Better Prognosis of Pulmonary Metastasectomy for Hepatocellular Carcinoma. Ann Thorac Surg 2019; 108: 1685-1691.

3. Uchino K, Tateishi R, Shiina S et al. Hepatocellular carcinoma with extrahepatic metastasis: clinical features and prognostic factors. Cancer 2011; 117: 4475-4483.

4. Abbas A, Medvedev S, Shores N et al. Epidemiology of metastatic hepatocellular carcinoma, a nationwide perspective. Dig Dis Sci 2014; 59: 2813-2820.

5. Ferlay J, Shin HR, Bray F et al. Estimates of worldwide burden of cancer in 2008: GLOBOCAN 2008. Int J Cancer 2010; 127: 2893-2917.

6. Mizuguchi S, Nishiyama N, Izumi N et al. Clinical Significance of Multiple Pulmonary Metastasectomy for Hepatocellular Carcinoma. World J Surg 2016; 40: 380-387.

7. Ye G, Wang L, Hu Z et al. Risk and prognostic nomograms for hepatocellular carcinoma with newlydiagnosed pulmonary metastasis using SEER data. PeerJ 2019; 7: e7496.

8. Invenizzi F, Iavarone M, Donato MF et al. Pulmonary Resection for Metastasis of Hepatocellular Carcinoma Recurring After Liver Transplant: An Italian Multicenter Experience. Front Oncol 2020; 10: 381.

9. Takahashi Y, Ikeda N, Nakajima J et al. Prognostic Analysis of Surgical Resection for Pulmonary Metastasis from Hepatocellular Carcinoma. World J Surg 2016; 40: 2178-2185.

10. Wu C, Ren X, Zhang Q. <p > Incidence, risk factors, and prognosis in patients with primary hepatocellular carcinoma and lung metastasis: a population-based study $</ \mathrm{p}>$. Cancer Management and Research 2019; Volume 11: 2759-2768.

11. Schmelzle M, Krenzien F, Schoning W, Pratschke J. Laparoscopic liver resection: indications, limitations, and economic aspects. Langenbecks Arch Surg 2020; 405: 725-735.

12. Lee DH, Kim D, Park YH et al. Long-term surgical outcomes in patients with hepatocellular carcinoma undergoing laparoscopic vs. open liver resection: A retrospective and propensity score-matched study. Asian J Surg 2020.

13. Solaini L, Bocchino A, Cucchetti A, Ercolani G. Anatomic Laparoscopic Liver Resection in the Scenario of the Hepatocellular Carcinoma: A Systematic Review and Meta-Analysis. J Laparoendosc Adv Surg Tech A 2020.

14. Wei Chieh AK, Chan A, Rotellar F, Kim KH. Laparoscopic major liver resections: Current standards. Int J Surg 2020. 